\title{
Surface-enhanced Raman scattering of self-assembled thiol monolayers and supported lipid membranes on thin anodic porous alumina
}

\author{
Marco Salerno ${ }^{* 1}$, Amirreza Shayganpour ${ }^{1,2}$, Barbara Salis ${ }^{1,2}$ and Silvia Dante ${ }^{1}$
}

\author{
Full Research Paper \\ Address: \\ ${ }^{1}$ Department of Nanophysics, Istituto Italiano di Tecnologia, via \\ Morego 30, I-16163 Genova, Italy and ${ }^{2}$ Department of Bioengineering \\ and Robotics, University of Genova, viale Causa 13, I-16145 Genova, \\ Italy \\ Email: \\ Marco Salerno* - marco.salerno@iit.it \\ * Corresponding author \\ Keywords: \\ anodic porous alumina; SERS; nanopores; supported lipid bilayers; \\ thiols
}

Beilstein J. Nanotechnol. 2017, 8, 74-81. doi:10.3762/bjnano.8.8

Received: 15 July 2016

Accepted: 12 December 2016

Published: 09 January 2017

This article is part of the Thematic Series "Self-assembly of nanostructures and nanomaterials II".

Guest Editor: I. Berbezier

(C) 2017 Salerno et al.; licensee Beilstein-Institut.

License and terms: see end of document.

\begin{abstract}
Thin anodic porous alumina (tAPA) was fabricated from a $500 \mathrm{~nm}$ thick aluminum (Al) layer coated on silicon wafers, through single-step anodization performed in a Teflon electrochemical cell in $0.4 \mathrm{M}$ aqueous phosphoric acid at $110 \mathrm{~V}$. Post-fabrication etching in the same acid allowed obtaining tAPA surfaces with $\approx 160 \mathrm{~nm}$ pore diameter and $\approx 80 \mathrm{~nm}$ corresponding wall thickness to be prepared. The tAPA surfaces were made SERS-active by coating with a thin $(\approx 25 \mathrm{~nm})$ gold (Au) layer. The as obtained tAPA-Au substrates were incubated first with different thiols, namely mercaptobenzoic acid (MbA) and aminothiol (AT), and then with phospholipid vesicles of different composition to form a supported lipid bilayer (SLB). At each step, the SERS substrate functionality was assessed, demonstrating acceptable enhancement $(\geq 100 \times)$. The chemisorption of thiols during the first step and the formation of SLB from the vesicles during the second step, were independently monitored by using a quartz crystal microbalance with dissipation monitoring (QCM-D) technique. The SLB membranes represent a simplified model system of the living cells membranes, which makes the successful observation of SERS on these films promising in view of the use of tAPA-Au substrates as a platform for the development of surface-enhanced Raman spectroscopy (SERS) biosensors on living cells. In the future, these tAPA-Au-SLB substrates will be investigated also for drug delivery of bioactive agents from the APA pores.
\end{abstract}

\section{Introduction}

Anodic porous alumina (APA) is a layered material usually obtained in thick form $(\approx 10 \mu \mathrm{m}$ thickness scale) from electrochemical anodization in the acidic aqueous electrolyte of aluminum (Al) foils [1]. In APA, the control of pore size, pore densi- ty and porosity is achieved by changing the anodization voltage during the fabrication and the etching parameters during the post-fabrication treatment [2]. It is widely recognized that the APA surface is biocompatible with practically all cell types and 
provides a means of controlling the surface roughness [3,4], the latter of which can play an important role in the adhesion and proliferation of cells [5-7]. The self-ordered nano-structured APA, also demonstrated recently as a possible nanolithographic mask [8,9] and for chemical sensors and biosensors [10], after coating with noble metals can be used for plasmonics-based enhanced spectroscopy such as in surface-enhanced Raman spectroscopy (SERS) [11-14].

In recent years, the thin form of APA (tAPA), resulting from anodization of $\mathrm{Al}$ films of less than $1 \mu \mathrm{m}$ thickness, has been increasingly used because it can be better integrated into applications involving optical microscopy inspection, which requires flat planar substrates. Moreover, it allows to move toward a more robust engineering of APA surfaces by exploiting the standard microtechnology of photolithography, thereby paving the way to large scale fabrication in possible future devices.

The enhancement factor in APA-based SERS can be as high as 1000 , which means that the technique may detect molecules [15]. Additionally, the pores in tAPA can potentially serve as nano-wells for localized drug delivery $[16,17]$. In fact, while lower in loading capacity with respect to thick APA [18], $500 \mathrm{~nm}$ tAPA can still allocate a significant amount of bioactive compounds, representing a trade-off between the former case of maximized loading and the case of ultra-thin APA showing the highest SERS enhancement [19]. Finally, the controlled roughness of APA could also improve the physisorption of coating layers of functional materials [20,21].

The main component of the biological membrane that separates and protects the interior of all living cells from the outside environment is a phospholipid bilayer. For this reason, as well as for the complexity of real samples of living cells, we decided to test the tAPA-Au SERS-active substrates on SLBs in phosphate-buffered saline (PBS) buffer solution, which provide an excellent model system to mimic the native cellular membranes [22].

In the present work, the fabrication and modification of tAPA aiming at its exploitation as a functional substrate for biosensing based on SERS effect are presented. In particular, it is reported on SERS effect on SLBs obtained from spontaneous lipid vesicle fusion and representing a simplified model of living cells membrane. Since the vesicle fusion is not trivial to achieve on Au surfaces, we first functionalized the Au with self-assembled monolayers (SAM) of thiols, to provide the appropriate surface condition to allow SLB formation. SERS effect was tested and proved for each fabrication step of the system.

\section{Experimental tAPA fabrication and modification to achieve SERS-activity}

An $\approx 500 \mathrm{~nm}$ thick Al layer was first coated on a silicon wafer by an electron-beam evaporation system PVD75 (Kurt J. Lesker Ltd., UK) working at a base pressure of $10^{-6}$ Torr with a deposition rate of $0.5-1 \AA / \mathrm{s}$. tAPA was fabricated in a single-step $(\approx 15 \mathrm{~min})$ anodization performed at $110 \mathrm{~V}$ in $0.4 \mathrm{M}$ phosphoric acid electrolyte at a bath temperature of $\approx 15{ }^{\circ} \mathrm{C}$. Post-fabrication etching in the same electrolyte for $20 \mathrm{~min}$ at room temperature (RT) plus $15 \mathrm{~min}$ at $35^{\circ} \mathrm{C}$ allowed to obtain tAPA with $\approx 160 \mathrm{~nm}$ pore size and $\approx 80 \mathrm{~nm}$ wall thickness. After thoroughly rinsing with de-ionized water, blowing dry with nitrogen and dehydrating on a hotplate set at $100{ }^{\circ} \mathrm{C}$ for $15 \mathrm{~min}$, the tAPA was overcoated by the same electron-beam evaporation system with $\mathrm{a} \approx 25 \mathrm{~nm}$ thick Au layer to make it SERS-active. More details on similar fabrication procedure can be found in references [12,13].

The characteristic size of tAPA pores was obtained by scanning electron microscope (SEM) imaging with a JSM-7500F (Jeol, Japan) and subsequent grain analysis carried out with Igor 6.22 (Wavemetrics, OR, USA).

\section{Incubation of thiols and fabrication of lipid vesicles}

Different thiols were used in combination with the different lipids to be coated onto them by electrostatically-driven physisorption. We used two thiols, namely 4-mercaptobenzoic acid (MbA) and 11-amino-1-undecanethiol hydrochloride (AT), from Sigma (Milan, Italy), and three lipids, namely 1-palmitoyl2-oleoyl-sn-glycero-3-phosphocholine (POPC), 1-palmitoyl-2oleoyl-sn-glycero-3-phospho-L-serine (POPS) and, 1,2dioleoyl-3-trimethylammoniumpropane (DOTAP), from Avanti Polar Lipids (Alabaster, Alabama, US). All solvents were purchased from Sigma-Aldrich.

First, the substrates were incubated at $\mathrm{rt}$ for $2 \mathrm{~h}$ with a $1 \mathrm{mM}$ aqueous solution of the thiol molecule, either MbA or AT, to let the sulfur of the - SH group bind covalently to the Au surface (chemisorption). The substrates were then gently washed with their aqueous solutions and dried under nitrogen flow.

All the lipids were dissolved in chloroform/methanol $2: 1 \mathrm{vol} / \mathrm{vol}$, dried under a gentle nitrogen flux in a test tube, and put under a mild vacuum overnight to remove all solvent traces. POPC/POPS in a 9:1 mol/mol ratio and DOTAP were then re-suspended in PBS at a $5 \mathrm{~g} / \mathrm{L}$ concentration, let to swell for $30 \mathrm{~min}$, and extruded 11 times through a polycarbonate filter (Whatman, USA) with $100 \mathrm{~nm}$ pore diameter to form unilamellar vesicles. 


\section{Preparation of the Raman target analytes: SLBs}

The lipid vesicles were diluted to $0.5 \mathrm{~g} / \mathrm{L}$ in the PBS buffer and vortexed immediately before use. The thiol SAM was incubated overnight with the lipid vesicle dispersion, to allow vesicle physisorption and fusion onto the substrate. The following day the samples were carefully washed with PBS three times to remove the exceeding vesicles.

For the cationic lipids (namely DOTAP), we used a thiolated molecule that presents a positively charged group at its end. This is $\mathrm{MbA}$, whose $\mathrm{COOH}$ group is protonated in PBS buffer to $\mathrm{COO}^{-}$. For the anionic lipid mixture (namely POPC/POPS) AT was used, which becomes positive in aqueous solution because of the terminal amino group.

\section{SERS measurements}

SERS measurements were performed with a micro-Raman spectrometer inVia (Renishaw, UK) equipped with the software program WiRE 3.2. We used for excitation a laser with $785 \mathrm{~nm}$ wavelength and $100 \mathrm{~mW}$ power, equipped for dispersion with a grating with 1200 grooves $/ \mathrm{mm}$. For detection microscope objectives with a magnification of $50 \times($ NA: 0.75$)$ and $60 \times$ (water immersion, NA: 1.0) were used. The spectra were collected in the $300-3200 \mathrm{~cm}^{-1}$ spectral range.

The SERS enhancement factor $\mathrm{G}$ achieved by employing tAPA-Au with respect to the flat $\mathrm{Au}$ on silicon substrate can be estimated by using a simple formula:

$$
G=\frac{P_{\text {Ref }} t_{\text {Ref }}}{P_{\text {SERS }} t_{\mathrm{SERS}}} \times \frac{A_{\mathrm{Ref}}}{A_{\mathrm{SERS}}} \times \frac{I_{\mathrm{SERS}}}{I_{\mathrm{Ref}}} .
$$

where $P, t, A$ and $I$ are laser power, accumulation time, active area for molecule adsorption and Raman intensity of the specific band, respectively [13]. The subscripts, SERS and Ref, indi- cate SERS and Raman measurements on tAPA-Au and on flat Au substrates, respectively.

\section{QCM-D characterization of adsorption}

A quartz microbalance Z500 (KSV Instruments, Finland) was used for the QCM-D experiments. Au coated AT-cut quartz crystals (QSense, Sweden) with a $5 \mathrm{MHz}$ fundamental resonance frequency were used. Before each experiment, the quartz sensor was first cleaned in a UV/Ozone ProCleaner (BioForce Nanoscience, US) for $10 \mathrm{~min}$, then washed with milli-Q (18.2 $\mathrm{M} \Omega \cdot \mathrm{cm}$ resistivity) water, dried under nitrogen flux and cleaned again for $10 \mathrm{~min}$ in the ozone cleaner.

The sensor was then mounted in the measurement chamber. The chamber was filled with proper buffer (aqueous solution for thiols, PBS for DOTAP vesicles and milli-Q water for POPC/POPS vesicles), and left to reach an equilibrium $(\approx 30 \mathrm{~min}$ ) before injecting the solution of interest. $3 \mathrm{~mL}$ of solution ( $1 \mathrm{mM}$ thiols in aqueous solution, and a concentration of $0.25 \mathrm{~g} / \mathrm{L}$ for both DOTAP in PBS and POPC/POPS in milli-Q) where then injected in the measurement chamber and left until the adsorption process reached an end (overnight measurement for thiols, $2 \mathrm{~h}$ for vesicles). The sensor was then rinsed with the proper buffer solution. If not stated otherwise, changes in frequency and dissipation of the seventh overtone $(35 \mathrm{MHz})$ are shown; all experiments were carried out at a temperature of $22{ }^{\circ} \mathrm{C}$.

\section{Results and Discussion SERS-active tAPA-Au substrates}

The control of the geometrical features of nanostructured substrates is of critical importance in SERS [23]. The SEM images reported in Figure 1 show the good control achieved in both mean pore size and its dispersion and prove the long range uniformity of the surfaces with the Au coating to make it plasmonic-active.
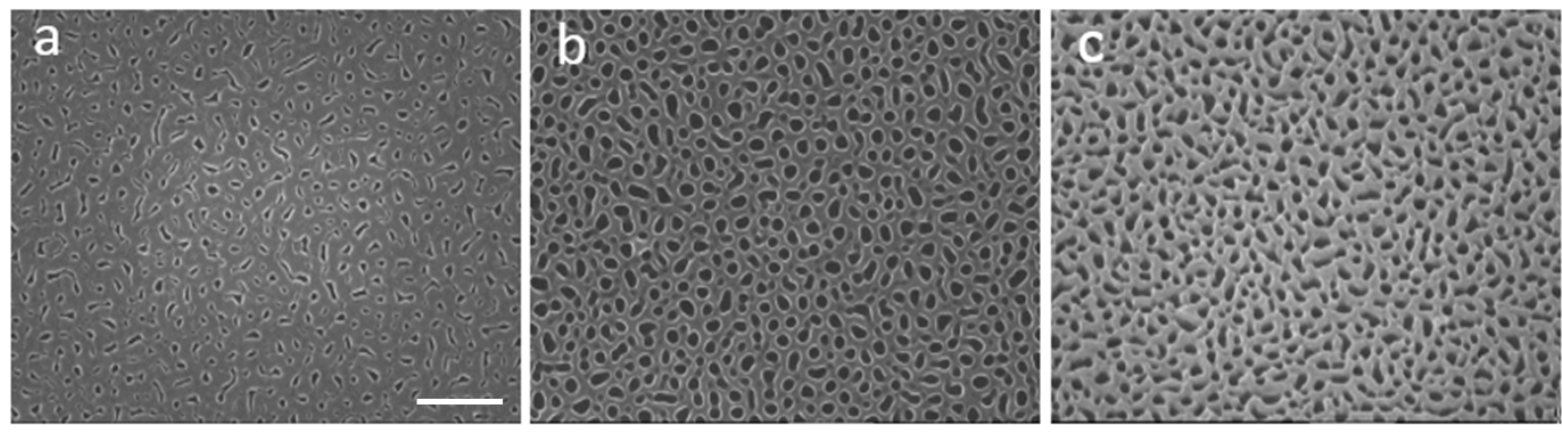

Figure 1: SEM images $(20,000 \times$ magnification, scale bar $1 \mu \mathrm{m})$ of tAPA substrates (thickness $\approx 500 \mathrm{~nm})$, a) as-prepared, b) after pore widening, and c) after $25 \mathrm{~nm}$ Au coating. 
tAPA-Au substrates could possibly be used as a carrier layer for local drug delivery [24], as a substrate for living cell cultures thanks to its controlled porosity [3-7], and for SERS [25]. However, since SERS is a surface-only effect, this sensitive detection will be limited to the top of the tAPA-Au substrates, i.e., to the bottom of the living cells, where they would adhere to the nanoporous substrate.

\section{SERS enhancement due to tAPA-Au on thiols, lipids, and thiol-lipid systems}

The Raman measurements were performed first on the thiol molecules. We started from the raw materials, in powder form, to obtain reference spectra for future comparison and best identification of the typical bands. Then, we measured the Raman scattering of the thiols adsorbed to flat Au substrates. For technical reasons of SLBs assembly, the two thiols selected, MbA and AT, in ethanol and PBS solutions have a negatively or positively charged group, respectively.

The spectra of the thiols powder on flat $\mathrm{Au}$, along with the respective molecular structures, are shown in Figure 2a. In
Figure $2 \mathrm{~b}$ the spectra of the SAM of the same thiols obtained after incubation on flat $\mathrm{Au}$ from $1 \mathrm{mM}$ water solution for $2 \mathrm{~h}$ at RT are shown. The subsequent step was the deposition of the thiol molecules for the formation of SAM onto tAPA-Au and the observation of the respective spectra. The nanopores in the oxide under the $\mathrm{Au}$, which are replicated by the top Au surface thanks to the low Au thickness of $\approx 25 \mathrm{~nm}$, allowed for SERS effect. In Figure 2c we report the typical Raman spectra obtained on $\mathrm{APPA}-\mathrm{Au}$ for both $\mathrm{MbA}$ and $\mathrm{AT}$.

The spectra of each thiol in all forms (pristine powder and film adsorbed onto the flat $\mathrm{Au}$ and tAPA-Au substrate) look similar. MbA present two major peaks at $\approx 1593$ and $\approx 1076 \mathrm{~cm}^{-1}$, which can be ascribed to aromatic ring vibrations, and also at $\approx 1181$ and at $\approx 1290 \mathrm{~cm}^{-1}$, which belong to $\mathrm{C}-\mathrm{H}$ mode [26-28]. AT presents the major peaks at $\approx 1434$ and $\approx 1477 \mathrm{~cm}^{-1}$ assigned to the $\mathrm{C}-\mathrm{H}$ and at $\approx 1074 \mathrm{~cm}^{-1}$ assigned to the $\mathrm{N}-\mathrm{H}$, while the peak at $\approx 1074 \mathrm{~cm}^{-1}$ belongs to the $\mathrm{C}-\mathrm{C}$ stretching.

In Supporting Information File 1, Figure S1 again the Raman spectra of both thiol SAMs, coupled according to the same thiol
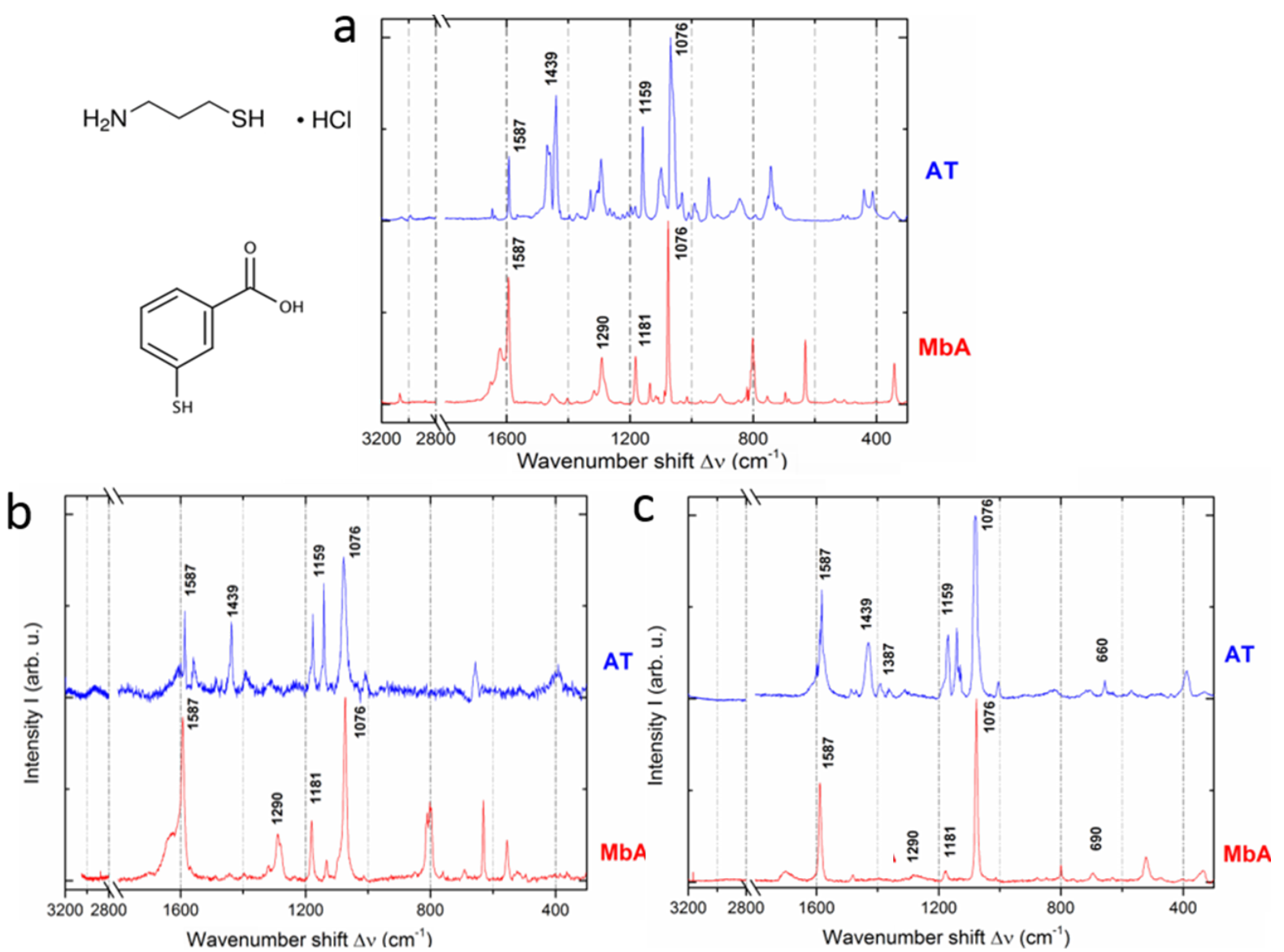

Figure 2: Raman spectra of thiols a) in powder form (with their molecular structures); b) in flat film form, after adsorption to the flat Au substrates from water solution; c) in film form on tAPA-Au. 
deposited on the different substrates of flat $\mathrm{Au}$ and tAPA-Au, are presented, for easier visualization of the substrate effect. It appears clearly that on tAPA-Au the major characteristic peaks of both $\mathrm{MbA}$ and $\mathrm{AT}$ are highly enhanced. Taking into account the measurement parameters (i.e., $t_{\text {Ref }}$ and $t_{\text {SERS }}$ both $10 \mathrm{~s}$, while $P_{\text {Ref }}$ and $P_{\text {SERS }}$ are 100 and 1 , respectively), a G factor of approximately 600 and 1000 was calculated for MbA and AT, respectively. The SERS effect of the nanophotonic tAPA structure, after coating with $\mathrm{Au}$ and thus thanks to the localized surface plasmon resonances of this thin film, emerges. The same effect may also be used on the SLBs, at the later stage of the model system fabrication.

For the selected lipids, we first tested the Raman spectra of the powders and then of the SLB form, on both the flat Au and nanoporous tAPA-Au. The molecular structure and Raman spectra of lipids in powder form are shown in Figure 3.

The molecules of choice, i.e., POPC and POPS, are two glycerophospholipids largely present in real cellular membranes. In particular, we prepared a mixture of POPC and POPS suspended in PBS with the molar ratio of $8: 2$, in order to resemble the plasma membrane composition both for charge and acyl chain length and unsaturation grade. However, the reason for the choice of DOTAP is technical, associated with the fabrication of artificial bilayer membranes [29,30].

The lipids are larger molecules than the thiols and present richer spectra, at least in the powder form. The main features in their Raman spectra depend on the hydrocarbon chain, and can be ascribed to scissoring and twisting of $\mathrm{CH}_{2}$ and $\mathrm{CH}_{3}$ and to stretching of $\mathrm{C}-\mathrm{C}$ and $\mathrm{C}-\mathrm{H}$. More precisely, the bands identi- fied in the higher wavenumber region appear at $3007 \mathrm{~cm}^{-1}$ (unsaturated $=\mathrm{CH}$ stretching), $2882 \mathrm{~cm}^{-1}\left(\mathrm{CH}_{2}\right.$ Fermi resonance) and $2847 \mathrm{~cm}^{-1}\left(\mathrm{CH}_{2}\right.$ symmetric stretching). The middle wavenumber region presents bands at $1737 \mathrm{~cm}^{-1}(\mathrm{C}=\mathrm{O}$ ester stretching), $1657 \mathrm{~cm}^{-1}(\mathrm{C}=\mathrm{C}$ stretching $), 1442 \mathrm{~cm}^{-1}\left(\mathrm{CH}_{2}\right.$ scissoring), $1300 \mathrm{~cm}^{-1}\left(\mathrm{CH}_{2}\right.$ twisting) and $1267 \mathrm{~cm}^{-1}(=\mathrm{C}-\mathrm{H}$ in-plane deformation). In the lower wavenumber region, the $\mathrm{C}-\mathrm{C}$ stretching emerges as a broad band around $1090 \mathrm{~cm}^{-1}$. In particular, two contributions at 1065 and $1089 \mathrm{~cm}^{-1}$ appear with a shoulder at $1125 \mathrm{~cm}^{-1}$. Additional bands appear at 719 and $876 \mathrm{~cm}^{-1}$ that are ascribed to the symmetric and asymmetric stretching of choline $\mathrm{N}^{+}\left(\mathrm{CH}_{3}\right)_{3}$, respectively [31,32].

The lipids were further investigated on tAPA-Au substrates, for the possible occurrence of SERS. Figure 4 shows Raman spectra for three mixtures of lipids in SLBs form, on both flat $\mathrm{Au}$ and tAPA-Au.

From the comparison of the spectra of lipids in SLBs form versus those in powder form, the most interesting difference observed is that in the films several peaks disappear or are weaker. Whereas some form of quenching can't be excluded, this is probably due to light polarization constraints in the ordered geometry of the molecular film, where not all modes of chemical groups may be excited, as it can be instead in the assembly of randomly oriented microcrystals of the powders [33].

It appears that on $\mathrm{APA}-\mathrm{Au}$ as compared to flat $\mathrm{Au}$ the characteristic thiol peaks are still present and enhanced. As a consequence, for $\mathrm{MbA}$ the major peaks at $\approx 1590$ and $\approx 1080 \mathrm{~cm}^{-1}$, ascribed to aromatic ring vibrations, and $\approx 1181 \mathrm{~cm}^{-1}$, ascribed to $\mathrm{C}-\mathrm{H}$ deformation, appear. Also AT presents the major peaks

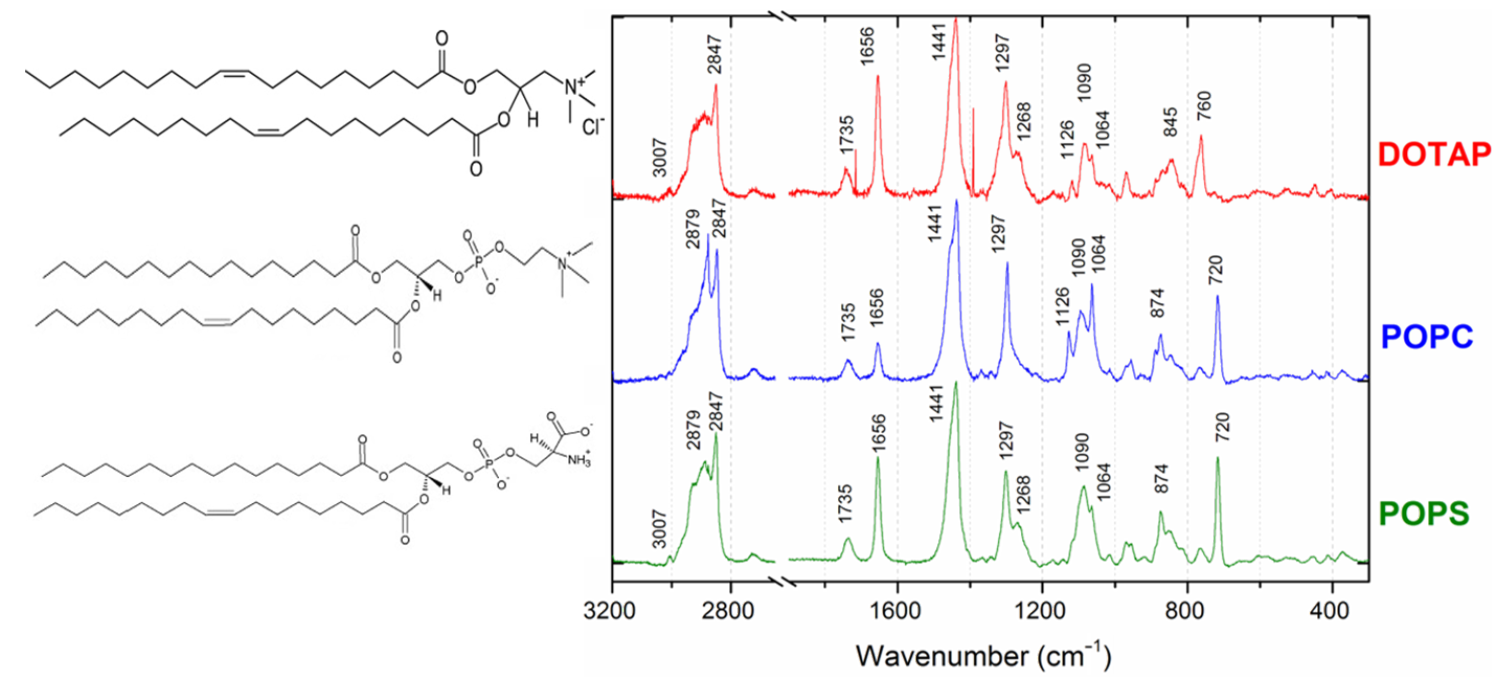

Figure 3: Raman spectra of lipids in powder form on flat Au substrates, with their molecular structures. 

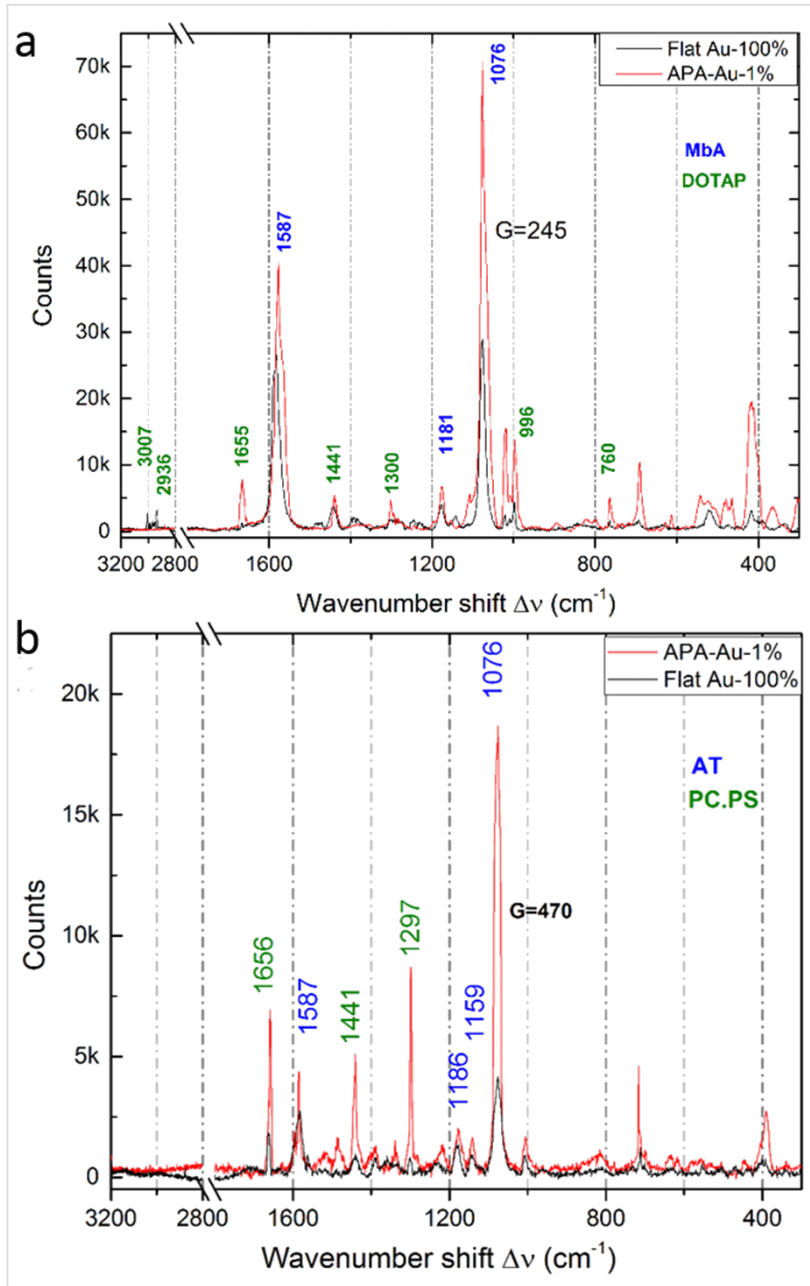

Figure 4: Raman spectra of the thiol-SLB systems on both flat $A u$ and tAPA-Au: a) MbA and DOTAP, b) AT and POPC/POPS blend.

at $\approx 1580, \approx 1159$ and $\approx 1074 \mathrm{~cm}^{-1}$, due to $\mathrm{C}-\mathrm{NH}, \mathrm{N}-\mathrm{H}$ wagging and $\mathrm{C}-\mathrm{C}$ stretching mode, respectively.

Additionally, in Figure 4 we have bands from the lipids, namely $\approx 1656 \mathrm{~cm}^{-1}$ ( $\mathrm{C}=\mathrm{C}$ stretching $), \approx 1440 \mathrm{~cm}^{-1}\left(\mathrm{CH}_{2}\right.$ scissoring $)$, $\approx 1300 \mathrm{~cm}^{-1}\left(\mathrm{CH}_{2}\right.$ twisting), $\approx 1267 \mathrm{~cm}^{-1}$ (=C-H in-plane deformation), and $\approx 719 \mathrm{~cm}^{-1}$ (choline) [34].

The values of enhancement $\mathrm{G}$ due to the tAPA-Au nanostructured substrates have been calculated according to Equation 1. Since the peaks on tAPA-Au are 2-4 times higher in the presence of 100 times lower laser power, a $\mathrm{G}$ of $\approx 250$ at $\approx 1076 \mathrm{~cm}^{-1}$ and 500 at $\approx 1076 \mathrm{~cm}^{-1}$ is obtained for the thiol-lipid system of MbA-DOTAP and AT-POPC/POPS, respectively.

\section{QCM-D measurements}

The lipid adsorption process on Au was independently monitored by QCM-D technique. This method allows the quantifica- tion of the adsorbed mass onto the surface of a vibrating $\mathrm{Au}$-coated quartz electrode through the measurement of the mass-induced frequency shift. Additionally, the measurement of the dissipation gives indication about the viscoelastic properties of the adsorbed layer. The quartz-Au substrate was thus used as a control for success of the incubation of the tAPA-Au substrates in the lipid dispersion. In a preliminary step (data not shown) we have monitored the chemisorption of thiols onto the Au-coated QCM-D sensors; the functionalized sensors where then exposed to the lipid vesicles and the process of adsorption was monitored.

The QCM-D time-evolution profiles presented in Figure 5a,b show that the lipids successfully adsorbed to the Au surface of quartz in both cases. However, the two lipid systems behave differently. For DOTAP on MbA (Figure 5a) one can observe a big shift in frequency $(\Delta f \approx-1135 \mathrm{~Hz})$ and a high value of dissipation $\left(D \approx 40 \times 10^{-6}\right)$, indicating the adsorption on the sensor's surface of a viscoelastic structure [35]. DOTAP vesicles do not fuse on Au functionalized with MbA, rather entire vesicles are adsorbed instead. On the contrary, for POPC/POPS on AT (Figure $5 \mathrm{~b}$ ) the frequency shift is low $(\Delta f \approx-157 \mathrm{~Hz}$ for the reported 7 th harmonic) and the value of dissipation is close to zero, indicating the adsorption of a smaller mass with more rigid structure on the surface. The reason may be that the POPC/POPS vesicles rupture in contact with the AT-functionalized $\mathrm{Au}$ and an SLB forms on the surface [36,37]. Table 1 shows the thickness values of SAM and adsorbed layers obtained with the QCM software. The values have been retrieved by using the Sauerbrey model of rigid layers for the SLBs and the Voigt model of viscoelastic layers for the adsorbed vesicles, assuming for the material densities the following values: $\rho_{\mathrm{AT}}=0.9 \mathrm{~g} / \mathrm{cm}^{3}$ for $\mathrm{AT}, \rho_{\mathrm{MbA}}=1.34 \mathrm{~g} / \mathrm{cm}^{3}$ for $\mathrm{MbA}$, and $\rho_{\mathrm{v}}=1 \mathrm{~g} / \mathrm{cm}^{3}$ for vesicles (made mostly of water), according to references $[38,39]$. The data confirm that DOTAP vesicles adsorb on the sensor without rupturing, with a thickness of the adsorbed layer of $\approx 90 \mathrm{~nm}$. POPC/POPS vesicles create an SLB on the sensor with a thickness of $\approx 4 \mathrm{~nm}$. When the formation of an SLB occurs, the fingerprint region is not visible. As already pointed out in Figure 4, we ascribe this effect to the orientation of the molecules and the polarization of the incoming beam. When vesicles are adsorbed on the surface, all the characteristic peaks of the lipid molecules are expected from the Raman spectra, since the vesicles contain all molecular orientations. In accordance to this, the Raman spectra of DOTAP collected from QCM sensor show a signal in the lipid fingerprint region which is different from the spectra collected on the Au-tAPA surface that are flat in the $2800-3000 \mathrm{~cm}^{-1}$ region. This indicates that the porosity of the substrate may influence the vesicle fusion process. This finding is still under further investigation. 

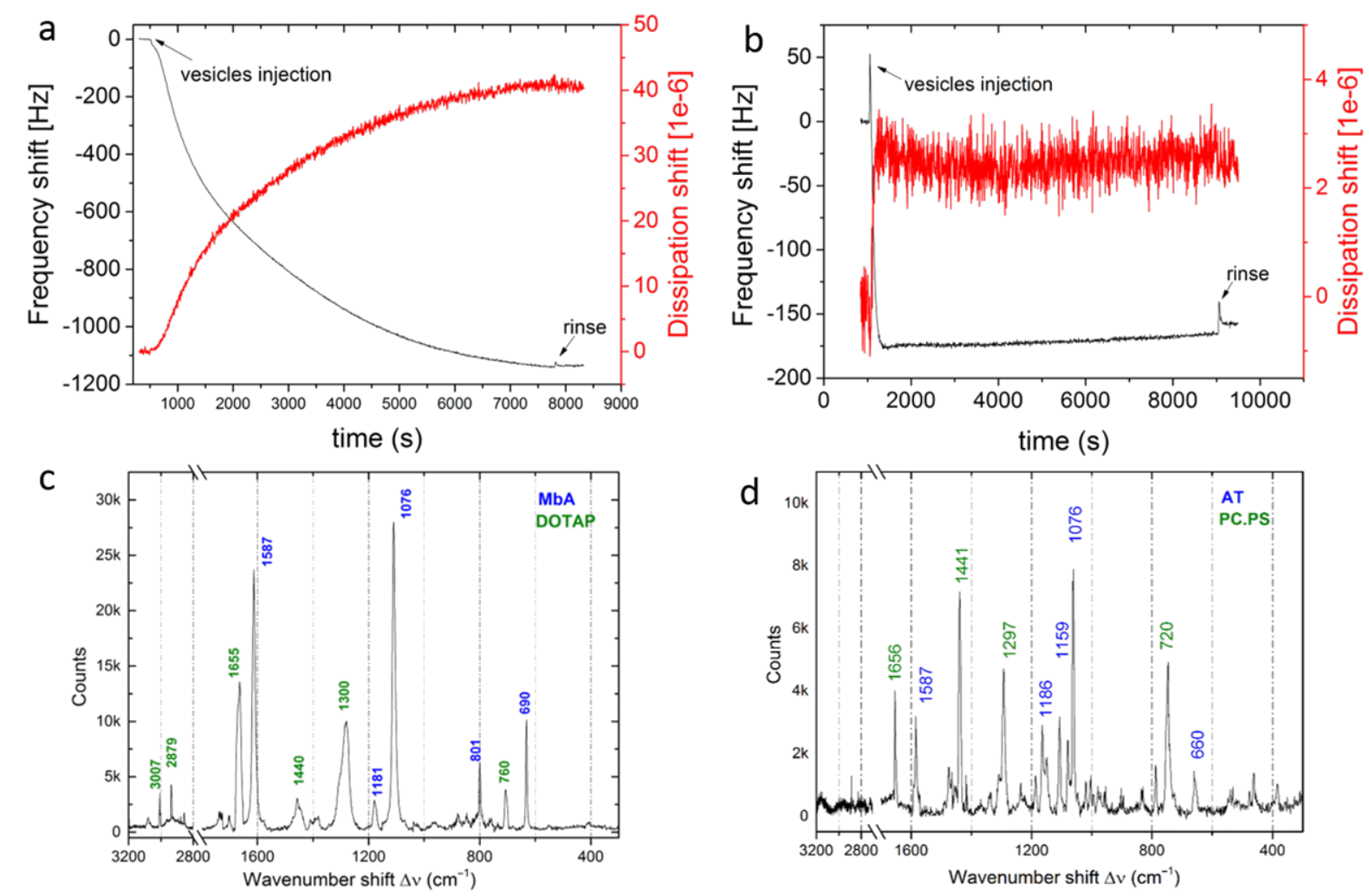

Figure 5: $a, b)$ QCM-D measurements of shift in frequency and dissipation of a) DOTAP on MbA substrate, and b) POPC/POPS on AT substrate; c,d) respective Raman spectra on QCM sensors coated with c) MbA and DOTAP, and d) AT and POPC/POPS blend.

Table 1: Shift in frequency $\Delta f$ and shift in dissipation $D$ and relative standard deviations of the layers adsorbed on the QCM sensor's surface. Every experiment was repeated three times.

\begin{tabular}{|c|c|c|c|c|c|c|}
\hline Solution & $d[\mathrm{~nm}]$ & std dev [nm] & $\Delta f[\mathrm{~Hz}]$ & std $\operatorname{dev}[\mathrm{Hz}]$ & $D\left[10^{-6}\right]$ & std dev $\left[10^{-6}\right]$ \\
\hline POPC/POPS (milli-Q) AT & 3.9 & 0.1 & -92 & 14 & 1.5 & 0.4 \\
\hline DOTAP (PBS) MbA & 96 & 3.5 & -1054 & 19.4 & 41.5 & 0.2 \\
\hline
\end{tabular}

In Supporting Information File, Figure S1 the spectra of both lipids are presented again, grouped according to the different types of substrates, which makes it possible to compare the effect of the substrate on the resulting spectra.

\section{Conclusion}

We successfully fabricated tAPA substrates on silicon wafer through anodization of $\approx 500 \mathrm{~nm}$ thickness and post-production etching, resulting in oxide films with pores of $\approx 160 \mathrm{~nm}$ size and $\approx 80 \mathrm{~nm}$ wall thickness. After coating with a $\approx 25 \mathrm{~nm}$ Au layer covering the tAPA features, our substrates become SERS-active and allow for an investigation of the chemical vibrations of molecules, as demonstrated by sensitive Raman measurements on bare thiols and on their combinations with lipid membranes, namely MbA with DOTAP and AT with POPC/POPS. The en- hancement factor was estimated to be 500 to 1000 on tAPA-Au with respect to the flat Au surface and to the silicon substrate. The chemisorption of thiols and lipids was confirmed on quartz$\mathrm{Au}$ by QCM-D technique. The present results point to the possible future use of the tAPA-Au surfaces as disposable substrates for assessing the cell functionality in biosensors/ bioassays.

\section{Supporting Information}

\section{Supporting Information File 1}

Additional figures.

[http://www.beilstein-journals.org/bjnano/content/ supplementary/2190-4286-8-8-S1.pdf] 


\section{References}

1. Diggle, J. W.; Downie, T. C.; Goulding, C. W. Chem. Rev. 1969, 69, 365-405. doi:10.1021/cr60259a005

2. Sulka, G. D. Nanostructured Materials in Electrochemistry; Wiley-VCH: Hoboken, NJ, U.S.A., 2008; pp 1-116.

3. Karlsson, M.; Johansson, A.; Tang, L.; Boman, M. Microsc. Res. Tech. 2004, 63, 259-265. doi:10.1002/jemt.20040

4. Salerno, M.; Caneva-Soumetz, F.; Pastorino, L.; Patra, N.; Diaspro, A.; Ruggiero, C. IEEE Trans. NanoBioscience 2013, 12, 106-111. doi:10.1109/TNB.2013.2257835

5. Gentile, F.; Tirinato, L.; Battista, E.; Causa, F.; Liberale, C.; di Fabrizio, E. M.; Decuzzi, P. Biomaterials 2010, 31, 7205-7212. doi:10.1016/j.biomaterials.2010.06.016

6. Ross, A. M.; Jiang, Z.; Bastmeyer, M.; Lahann, J. Small 2012, 8, 336-355. doi:10.1002/smll.201100934

7. Rahmany, M. B.; Van Dyke, M. Acta Biomater. 2013, 9, 5431-5437. doi:10.1016/j.actbio.2012.11.019

8. Yanagishita, T.; Masuda, H. AIP Adv. 2016, 6.

9. Yanagishita, T.; Masuda, H. Electrochim. Acta 2015, 184, 80-85. doi:10.1016/j.electacta.2015.10.019

10. Santos, A.; Kumeria, T.; Losic, D. TrAC, Trends Anal. Chem. 2013, 44, 25-38. doi:10.1016/j.trac.2012.11.007

11. Chung, A. J.; Huh, Y. S.; Erickson, D. Nanoscale 2011, 3, 2903-2908. doi:10.1039/c1nr10265f

12. Toccafondi, C.; Thorat, S.; La Rocca, R.; Scarpellini, A.; Salerno, M.; Dante, S.; Das, G. J. Mater. Sci.: Mater. Med. 2014, 25, 2411-2420. doi:10.1007/s10856-014-5178-4

13. Toccafondi, C.; La Rocca, R.; Scarpellini, A.; Salerno, M.; Das, G.; Dante, S. Appl. Surf. Sci. 2015, 351, 738-745. doi:10.1016/j.apsusc.2015.05.169

14. Kneipp, K.; Kneipp, H.; Kneipp, J. Acc. Chem. Res. 2006, 39, 443-450. doi:10.1021/ar050107x

15. Nie, S.; Emory, S. E. Science 1997, 275, 1102-1106. doi:10.1126/science.275.5303.1102

16. Salerno, M.; Thorat, S. 13th Ceram. Cells Tissues. Regen. nanomedicine, tissue Genet. Eng. role Ceram. 2011, 171.

17. Gultepe, E.; Nagesha, D.; Sridhar, S.; Amiji, M. Adv. Drug Delivery Rev. 2010, 62, 305-315. doi:10.1016/j.addr.2009.11.003

18. Aw, M. S.; Bariana, M.; Losic, D. Nanoporous Anodic Alumina for Drug Delivery and Biomedical Applications. In Nanoporous Alumina: Fabrication, Structure, Properties and Applications; Losic, D.; Santos, A., Eds.; Springer Series in Materials Science, Vol. 219; Springer: Berlin, Germany, 2015; pp 319-354. doi:10.1007/978-3-319-20334-8_11

19. Toccafondi, C.; Zaccaria, R. P.; Dante, S.; Salerno, M. Materials 2016, 9, 403-414. doi:10.3390/ma9060403

20. Swan, E. E. L.; Popat, K. C.; Desai, T. A. Biomaterials 2005, 26, 1969-1976. doi:10.1016/j.biomaterials.2004.07.001

21. Chang, P.-C.; Liu, B.-Y.; Liu, C.-M.; Chou, H.-H.; Ho, M.-H.; Liu, H.-C.; Wang, D.-M.; Hou, L.-T. J. Biomed. Mater. Res., Part A 2007, 81, 771-780. doi:10.1002/jbm.a.31031

22. Tamm, L. K.; McConnell, H. M. Biophys. J. 1985, 47, 105-113. doi:10.1016/S0006-3495(85)83882-0

23. Das, G.; Patra, N.; Gopalakrishnan, A.; Zaccaria, R. P.; Toma, A.; Thorat, S.; Di Fabrizio, E.; Diaspro, A.; Salerno, M. Analyst 2012, 137, 1785. doi:10.1039/c2an16022f

24. Thorat, S.; Diaspro, A.; Scarpellini, A.; Povia, M.; Salerno, M. Materials 2013, 6, 206-216. doi:10.3390/ma6010206
25. Das, G.; Patra, N.; Gopalakrishanan, A.; Proietti Zaccaria, R.; Toma, A.; Thorat, S.; Di Fabrizio, E.; Diaspro, A.; Salerno, M. Microelectron. Eng. 2012, 97, 383-386. doi:10.1016/j.mee.2012.02.037

26. Zheng, J.; Zhou, Y.; Li, X.; Ji, Y.; Lu, T.; Gu, R. Langmuir 2003, 19, 632-636. doi:10.1021/la011706p

27. Mayo, D. W.; Miller, F. A.; Hannah, R. W. In Course Notes on the Interpretation of Infrared and Raman Spectra; Mayo, D. W.; Miller, F. A.; Hannah, R. W., Eds.; Wiley: Hoboken, NJ, U.S.A., 2004; i-xxvi. doi:10.1002/0471690082.fmatter

28. Velleman, L.; Bruneel, J.-L.; Guillaume, F.; Losic, D.; Shapter, J. G. Phys. Chem. Chem. Phys. 2011, 13, 19587-19593. doi:10.1039/c1cp21765h

29. An, H. H.; Han, W. B.; Kim, Y.; Kim, H.-S.; Oh, Y.; Yoon, C. S. J. Raman Spectrosc. 2014, 45, 292-298. doi:10.1002/jrs.4461

30. Taylor, R. W.; Benz, F.; Sigle, D. O.; Bowman, R. W.; Bao, P.; Roth, J. S.; Heath, G. R.; Evans, S. D.; Baumberg, J. J. Sci. Rep. 2014, 4, 5940. doi:10.1038/srep05940

31. Schulz, H.; Baranska, M. Vib. Spectrosc. 2007, 43, 13-25. doi:10.1016/j.vibspec.2006.06.001

32. Köhler, M.; MacHill, S.; Salzer, R.; Krafft, C. Anal. Bioanal. Chem. 2009, 393, 1513-1520. doi:10.1007/s00216-008-2592-9

33. Zumbusch, A.; Langbein, W.; Borri, P. Prog. Lipid Res. 2013, 52, 615-632. doi:10.1016/j.plipres.2013.07.003

34. Czamara, K.; Majzner, K.; Pacia, M. Z.; Kochan, K.; Kaczor, A.; Baranska, M. J. Raman Spectrosc. 2015, 46, 4-20. doi:10.1002/jrs.4607

35. Richter, R. P.; Bérat, R.; Brisson, A. R. Langmuir 2006, 22, 3497-3505. doi:10.1021/la052687c

36. Reimhult, E.; Höök, F.; Kasemo, B. Langmuir 2003, 19, 1681-1691. doi:10.1021/la0263920

37. Peng, P.-Y.; Chiang, P.-C.; Chao, L. Langmuir 2015, 31, 3904-3911. doi:10.1021/la504532a

38. Campiña, J. M.; Martins, A.; Silva, F. J. Phys. Chem. C 2007, 111, 5351-5362. doi:10.1021/jp0662146

39. Hewas, A. M. Analysis of metal ions in water using SAM-modified EQCM electrodes. Ph.D. Thesis, Department of Chemistry, University of Leicester, 2013.

\section{License and Terms}

This is an Open Access article under the terms of the Creative Commons Attribution License (http://creativecommons.org/licenses/by/4.0), which permits unrestricted use, distribution, and reproduction in any medium, provided the original work is properly cited.

The license is subject to the Beilstein Journal of Nanotechnology terms and conditions: (http://www.beilstein-journals.org/bjnano)

The definitive version of this article is the electronic one which can be found at: doi: $10.3762 /$ bjnano.8.8 